\title{
Correction to: Intra-arterial Stem Cell Therapy Diminishes Inflammasome Activation After Ischemic Stroke: a Possible Role of Acid Sensing lon Channel 1a
}

\author{
Kanchan Vats ${ }^{1}$. Deepaneeta Sarmah ${ }^{1} \cdot$ Aishika Datta $^{1}$. Jackson Saraf ${ }^{1} \cdot$ Harpreet Kaur $^{1} \cdot$ Kanta Pravalika $^{1}$. \\ Madhuri Wanve $^{1} \cdot$ Kiran Kalia $^{1} \cdot$ Anupom Borah $^{2} \cdot$ Kunjan R. Dave $^{3} \cdot$ Dileep R. Yavagal $^{4} \cdot$ Pallab Bhattacharya $^{1}(1)$
}

Published online: 1 January 2021

(c) Springer Science+Business Media, LLC, part of Springer Nature 2020

\section{Correction to: Journal of Molecular Neuroscience https://doi.org/10.1007/s12031-019-01460-3}

It has come to our notice that there was an inadvertent misupload of Fig. 2 c, d in the loading control (GAPDH) for ASIC1a and NLRP1 blots. We would like to replace the same with the correct image. This change anyhow does not affect the conclusion of the study. However, the use of GAPDH as a loading control for stroke studies sometimes is debatable (Zhai et al. 2014; Kang et al. 2018). Hence, we repeated our experiments to check the expression of ASIC1a and NLRP1 at different time points following stroke, using beta actin as a loading control. We found that at $24 \mathrm{~h}$ post stroke, maximal and significant expression of both ASIC1a and NLRP1 was observed (Fig. 2 e, f). The expression at 48 and $72 \mathrm{~h}$ post stroke were not significantly different as compared to that of sham. The expression results obtained using beta actin as a loading control were concurrent with the previous results published with GAPDH as a loading control.

The original article can be found online at https://doi.org/10.1007/ s12031-019-01460-3

Pallab Bhattacharya

pallab.bhu@gmail.com; pallab.bhattacharya@niperahm.ac.in

1 Department of Pharmacology and Toxicology, National Institute of Pharmaceutical Education and Research (NIPER), Ahmedabad, Gandhinagar, Gujarat, India

2 Cellular and Molecular Neurobiology Laboratory, Department of Life Science and Bioinformatics, Assam University, Silchar, Assam, India

3 Department of Neurology, University of Miami Miller School of Medicine, Miami, FL, USA

4 Department of Neurology and Neurosurgery, University of Miami Miller School of Medicine, Miami, FL, USA 
a)

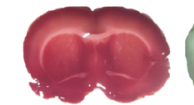

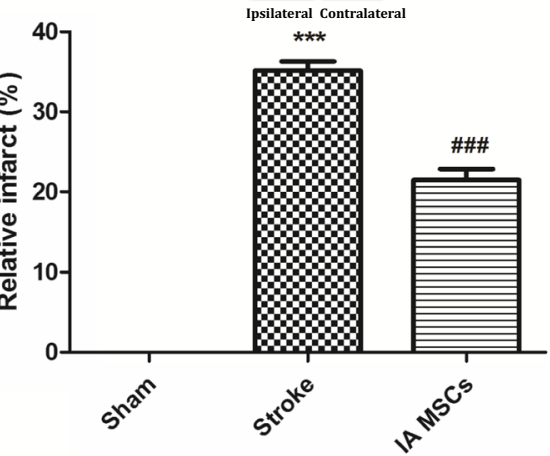

b)

LASER DOPPLER FLOWMETRY (LDF)

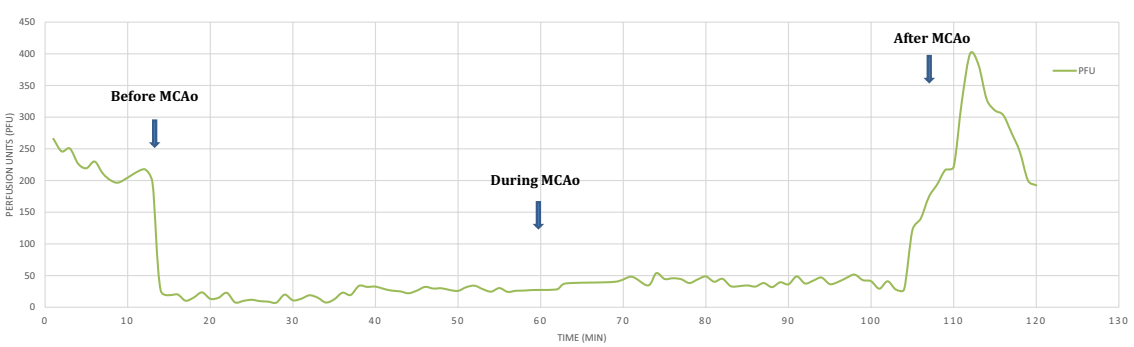

c)

ASIC1a

NLRP1

GAPDH

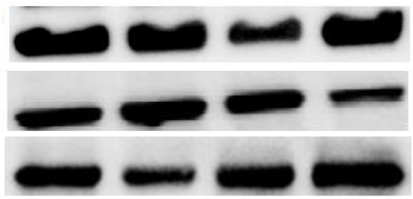

d)
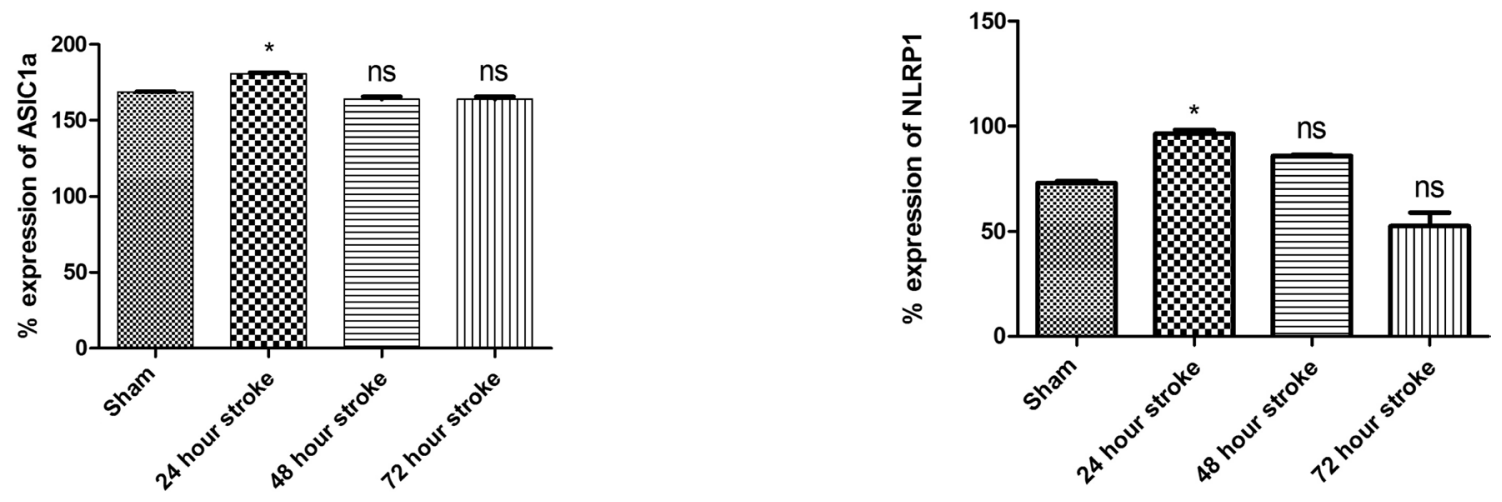

e)

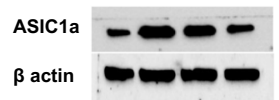

f)

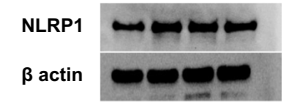

ASIC1a
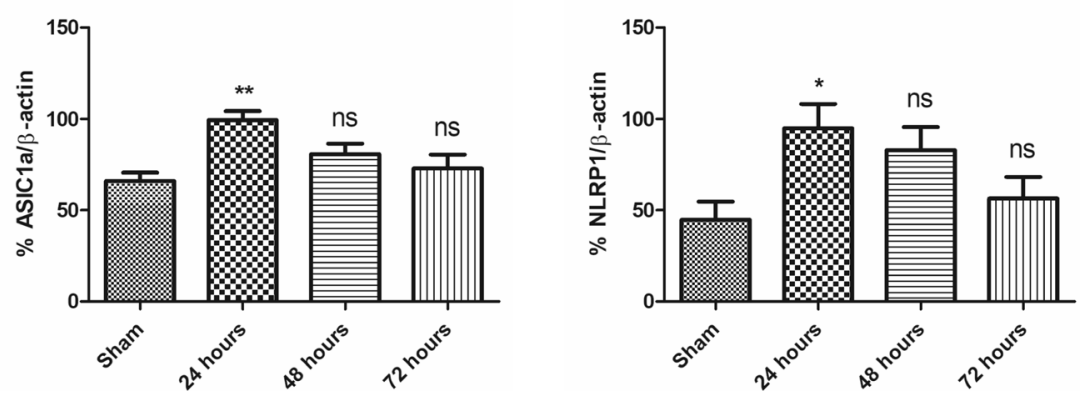

Fig. 2 Immunoreactive bands showing the expression of e) ASIC1a and f) NLRP1 at different time points following stroke (*v/s sham, $\mathrm{p} \leq 0.05$; $* * \mathrm{v} / \mathrm{s}$ sham, $\mathrm{p} \leq 0.01$ ) 


\section{References}

Zhai D, Chin K, Wang M, Liu F (2014) Disruption of the nuclear p53-GAPDH complex protects against ischemia-induced neuronal damage. Molecular brain 7(1):1-12

Kang Y, Wu Z, Cai D, Lu B (2018) Evaluation of reference genes for gene expression studies in mouse and $\mathrm{N} 2 \mathrm{a}$ cell ischemic stroke models using quantitative real-time PCR. BMC neuroscience 19(1):1-11
Publisher's Note Springer Nature remains neutral with regard to jurisdictional claims in published maps and institutional affiliations. 